\title{
Are wearable insoles a validated tool for quantifying transfemoral amputee gait asymmetry?
}

\author{
Isabelle Loiret' ${ }^{\text {ID, }}$, Coralie Villa ${ }^{2,3}$, Boris Dauriac ${ }^{2,4}$, \\ Xavier Bonnet ${ }^{2}$, Noël Martinet', Jean Paysant' ${ }^{1}$ and Hélène Pillet $^{2}$
}

\begin{abstract}
Background: Amputee gait is known to be asymmetrical, especially during loading of the lower limb. Monitoring asymmetry could be useful in quantifying patient performance during rehabilitation. Wearable insoles can provide normal ground reaction force asymmetry in real-life conditions.

Objectives: To characterize the validity of Loadsol ${ }^{\circledR}$ insoles versus force plates in quantifying normal ground reaction force and gait asymmetry. To determine the influence walking speed has on loading asymmetry in transfemoral amputees.

Study design: This is a prospective study.

Methods: Six transfemoral amputees, wearing Loadsol ${ }^{\circledR}$ insoles, walked at three self-selected speeds on force plates. Validity was assessed by comparing normal ground reaction force data from the insoles and force plates. The Absolute Symmetry Index was used to calculate gait loading asymmetry at each speed.

Results: Normalized root mean square errors for the normal ground reaction forces were $6.6 \%$ (standard deviation $=2.3 \%$ ) and $8.9 \%$ (standard deviation $=3.8 \%$ ); correlation coefficients were 0.91 and 0.95 for the prosthetic and intact limb, respectively. The mean error for Absolute Symmetry Index parameters ranged from $-2.67 \%$ to $4.35 \%$. Loading asymmetry increased with walking speed.

Conclusion: This study quantified the validity of Loadsol ${ }^{\circledR}$ insoles in assessing loading asymmetry during gait in transfemoral amputees. The calibration protocol could be improved to better integrate it into a clinical setting. However, our results support the relevance of using such insoles during the clinical follow-up of transfemoral amputees.
\end{abstract}

\section{Clinical relevance}

This is the first study to validate Loadso ${ }^{\circledR}$ insoles versus force plates and report on loading asymmetry during gait at three different speeds in transfemoral amputees. Loadsol ${ }^{\circledR}$ insoles, which provide visual and audio feedback, are clinically easy to use and could have beneficial application in the amputee's rehabilitation and follow-up.

\section{Keywords}

Validity, wearable insoles, transfemoral amputee, loading asymmetry, locomotion

\section{Background}

Walking with a prosthesis is challenging, particularly when the amputation is proximal to the knee joint. To cope with the loss of functionality of prosthetic joints compared to physiologic joints, people with lower limb amputations often modify the kinematics of their contralateral or residual limbs, which results in gait deviations, such as vaulting or pelvic hiking. ${ }^{1-4}$ These deviations could be quantified through biomechanical parameters derived from motion capture systems, but the use of such techniques in a clinical environment cannot be considered due to the complexity
'Centre de médecine physique et de réadaptation Louis Pierquin, IRR-UGECAM, Nancy Cedex, France

Institut de Biomécanique Humaine Georges Charpak, Arts et Metiers ParisTech, Paris, France

${ }^{3}$ Centre d'Etude et de Recherche sur l'Appareillage des Handicapés, INI, Woippy Cédex, France

${ }^{4}$ Handicap Technologie, PROTEOR, Seurre, France

Corresponding author:

Isabelle Loiret, Centre de médecine physique et de réadaptation

Louis Pierquin, IRR-UGECAM, Nord-Est, 75 Boulevard Lobau,

Nancy Cedex 54042, France.

Email: isabelle.loiretpro@gmail.com 
Table I. Demographic characteristics of the TFA (transfemoral amputee) group.

\begin{tabular}{|c|c|c|c|c|c|c|c|c|c|}
\hline & $\begin{array}{l}\text { Age } \\
\text { (years) }\end{array}$ & Gender & $\begin{array}{l}\text { Amputated } \\
\text { side }\end{array}$ & Etiology & $\begin{array}{l}\text { Time since } \\
\text { amputation } \\
\text { (years) }\end{array}$ & $\begin{array}{l}\text { Prosthetic } \\
\text { foot }\end{array}$ & $\begin{array}{l}\text { Prosthetic } \\
\text { knee }\end{array}$ & $\begin{array}{l}\text { Height } \\
\text { (cm) }\end{array}$ & $\begin{array}{l}\text { Mass } \\
(\mathbf{k g})\end{array}$ \\
\hline TFAOI & 47 & Female & Right & Tumor & 35 & Variflex $L^{\circledR}$ & RheoKnee ${ }^{\circledR}$ & 154 & 72.8 \\
\hline TFA02 & 52 & Male & Right & Trauma & 34 & Variflex $X C^{\circledR}$ & RheoKnee $^{\circledR}$ & 169 & 75.2 \\
\hline TFA03 & 34 & Female & Left & Tumor & 27 & Trias $^{\circledR}$ & C Leg ${ }^{\circledR}$ & 170 & 50.8 \\
\hline TFA04 & 39 & Male & Left & Trauma & 3 & Triton $^{\circledR}$ & C Leg ${ }^{\circledR}$ & 179 & 84.8 \\
\hline TFA05 & 25 & Male & Left & Trauma & 1.5 & Proflex ${ }^{\circledR}$ & C Leg ${ }^{\circledR}$ & 180 & 56 \\
\hline TFA06 & 32 & Male & Left & Trauma & 7 & Proflex ${ }^{\circledR}$ & RheoKnee $^{\circledR}$ & 180 & 95 \\
\hline
\end{tabular}

The prosthetic devices are from Ottobock ${ }^{\circledR}: \mathrm{Cleg}^{\circledR}$, Triton ${ }^{\circledR}$, and Trias $^{\circledR}$ and from Ossür ${ }^{\circledR}$ : Rheoknee ${ }^{\circledR}$, Variflex LP $^{\circledR}$, and Variflex XC ${ }^{\circledR}$.

of the instrumentation and processing required. Thus, some authors have focused on analyzing the impact of gait deviations on global parameters such as gait asymmetry between prosthetic and contralateral limbs. The asymmetry of spatio-temporal or kinematic parameters has been quantified for both transtibial and transfemoral amputees (TFAs). ${ }^{5-7}$ Recently, Cutti et al. ${ }^{8}$ and RutkowskaKucharska et al. ${ }^{9}$ have focused on the asymmetry of ground reaction forces and have given reference values for people with amputations. In addition, this asymmetry has been shown to be related to long-term pain such as osteoarthritis of the knee and hip joints, with prevalence rates of $27 \%$ and $14 \%$, respectively, which is significantly higher than the rates observed in the general population ${ }^{10}$ or individuals with osteopenia and osteoporosis. ${ }^{11}$ These studies confirmed the clinical relevance of asymmetry quantification in this population.

However, to be relevant in the clinical environment, asymmetry must be quantified by validated, low-cost devices. Pressure insoles appear to be an excellent option to address this task as they can measure the pressure under both feet with minimal instrumentation. Two studies have already conducted this analysis using $\mathrm{Pedar}^{\circledR 8}$ and $\mathrm{CDG}^{\circledR}$ sensor shoes. ${ }^{6}$ In addition, the validity and between-day repeatability have been investigated for other systems ${ }^{12-16}$ used in various pathologies: diabetes, ${ }_{17}^{17}$ ankle osteoarthritis, ${ }^{18}$ foot deformities, and flat foot. ${ }^{19}$ However, none of the above-mentioned devices were specifically designed for long-term use in real-life conditions, nor are they affordable for clinical use during the rehabilitation process.

Loadsol ${ }^{\circledR}$ insoles have recently been introduced by Novel (Munich, Germany) to overcome the limits of existing pressure insoles. These insoles are made with a single capacitive force sensor covering the whole surface of the foot. This new device directly measures the resulting foot load without post-processing of pressure data. With a mobile app on a smartphone, audio or vibratory feedback can be obtained in real time. Therefore, by providing the normal ground reaction force ( $\mathrm{NGrF}$ ) applied on each lower limb, this system could have a beneficial application in the rehabilitation and follow-up of lower limb amputees to correct gait asymmetry during loading.
However, to our knowledge, no study has validated the use of Loadsol ${ }^{\circledR}$ insoles in an amputee population. The first aim of the study was to measure the accuracy of Loadsol ${ }^{\circledR}$ insoles in quantifying $\mathrm{NGrF}$ compared to force plates (considered the gold standard) during gait in a population of individuals with a transfemoral amputation. The second aim was to supplement the existing data on gait asymmetry in this population by focusing on the influence of walking speeds on level ground, which has only been partly described previously. ${ }^{6}$

\section{Methods}

\section{Participants}

This prospective research was approved by the local ethics committee (CPP IDF VI, No. 2014-A01938-39). All participants gave their written informed consent prior to study enrolment. Participants with a unilateral transfemoral amputation due to trauma or a tumor $(n=6$, mean height: $172 \pm 10 \mathrm{~cm}$, mean mass: $75.5 \pm 27.6 \mathrm{~kg}$, mean age: $38 \pm 10$ years, time since amputation: $18 \pm 16$ years) were included (Table 1). Participants wore a comfortably fitted prosthesis and were able to walk without any assistive device at various walking speeds. Participants were excluded if they had any pathology or prosthetic limitations causing pain.

\section{Material}

Loadsol ${ }^{\circledR}$ insoles (Novel, Gmbh, Munich, Germany) (Figure 2) were placed in the participants' shoes. Composed of a single capacitive force sensor, each insole was able to record and/or visualize $\mathrm{NGrF}$ applied by the foot in the shoe in real time on a smartphone via a Bluetooth connection. Measurements were sampled at $100 \mathrm{~Hz}$.

\section{Experimental protocol}

Each participant walked at three self-selected speeds with the Loadsol ${ }^{\circledR}$ insoles in their own shoes (comfortable speed (self selected speed (SSS)), low self selected speed LSS), 
and fast self-selected speed (FSS)) along an 8-m-level walkway in a motion capture laboratory equipped with an optoelectronic system (VICON ${ }^{\circledR}$ V8i, $100 \mathrm{~Hz}$ ) and three force plates $\left(\mathrm{AMTI}^{\circledR}, 1000 \mathrm{~Hz}\right)$. Participants were required to complete a 15-min warm-up period with the insoles in their shoes. The insoles were then calibrated for recording. The calibration protocol corresponded to the manufacturer's recommendations, loading each lower limb alternatively with full body weight without the support of the upper limbs. A minimum of three trials were performed for each participant and each speed condition.

\section{Data analysis}

Data analysis was divided into two parts: validation of the Loadsol ${ }^{\circledR}$ insoles compared to the force plates only during trials at SSS and quantification of asymmetry based on insole data during trials at SSS, LSS, and FSS.

Loadso ${ }^{\circledR}$ validation. ASCII insole data and force plate data were post-processed using MATLAB ${ }^{\circledR}$. To synchronize the insoles and force plates, the first $\mathrm{NGrF}$ value above the threshold of $20 \mathrm{~N}$ for both measuring devices was taken as the first instance of ground contact, and force plate data were resampled at $100 \mathrm{~Hz}$ to correspond to the frequency of the insoles. The steps simultaneously recorded by both insoles and force platforms were extracted based on the SSS trial data.

Changes in NGrF over the cycle and four specific parameters (Figure 1), considered to be clinically relevant, were compared for each lower limb (intact and prosthetic) with both the insoles and force plates:

1. Magnitude of weight acceptance peak force (Fz1) in Newton (N);

2. Magnitude of the second peak force or push-off peak (Fz2) in Newton (N);

3. Force time integral (impulse) in Newton second (Ns);

4. Stance phase duration (time from heel strike to toe off) in seconds (s).

The Absolute Symmetry Index (ASI) was used to calculate all the parameters specified above according to the definition proposed by Nolan et al. ${ }^{6}$ The ASI can be used to quantify the asymmetry between the limbs and is defined in an amputee population using the following formula

$$
A S I=\frac{(I-P)}{0.5(I+P)} \times 100
$$

where $I$ is the intact limb and $P$ is the prosthetic limb. A positive value indicates that the considered parameter is higher for the intact limb than for the prosthetic limb.

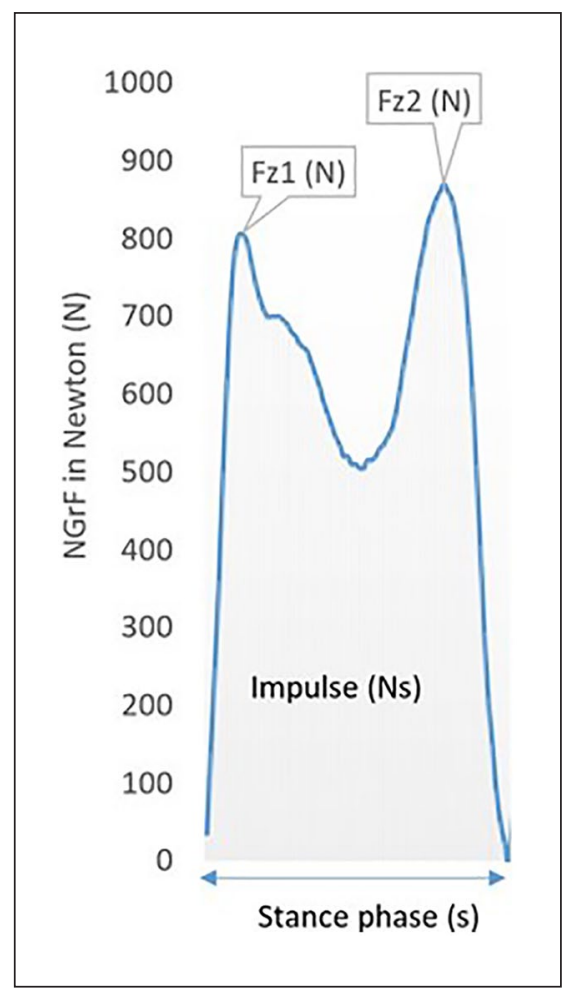

Figure I. Typical NGrF curve with specific parameters indicated.

Fzl: magnitude of weight acceptance peak force (N); Fz2: magnitude of the second peak force $(\mathrm{N})$; impulse: force time integral $(\mathrm{Ns})$; stance phase: time from heel strike to toe off (s).

The root mean square error (RMSE), normalized root mean square error (NRMSE), Bland-Altman plots, ${ }^{20}$ and Pearson's coefficient were calculated to compare changes in NGrF over the entire gait cycle for each lower limb (intact and prosthetic). Bland-Altman plots ${ }^{20}$ were drawn for the specific parameters and corresponding ASI values for each lower limb.

Gait asymmetry quantification. To assess gait asymmetry and its relationship with walking speed, six to nine gait cycles recorded with the insoles were used. The first and the last steps of each trial were removed. ASI values for the specific parameters were calculated for each subject and the three walking speed conditions. Cadence was expressed in strides per minute.

For each ASI value, Friedman's analysis of variance (ANOVA) was used to detect statistical differences between the three walking speed conditions with a significance level of 0.05 . Considering the non-normal distribution of data, a post hoc non-parametric Wilcoxon test with a Bonferroni correction was then performed to test statistical differences using a side-by-side comparison of speed conditions. The alpha level was decreased to 0.016 . 


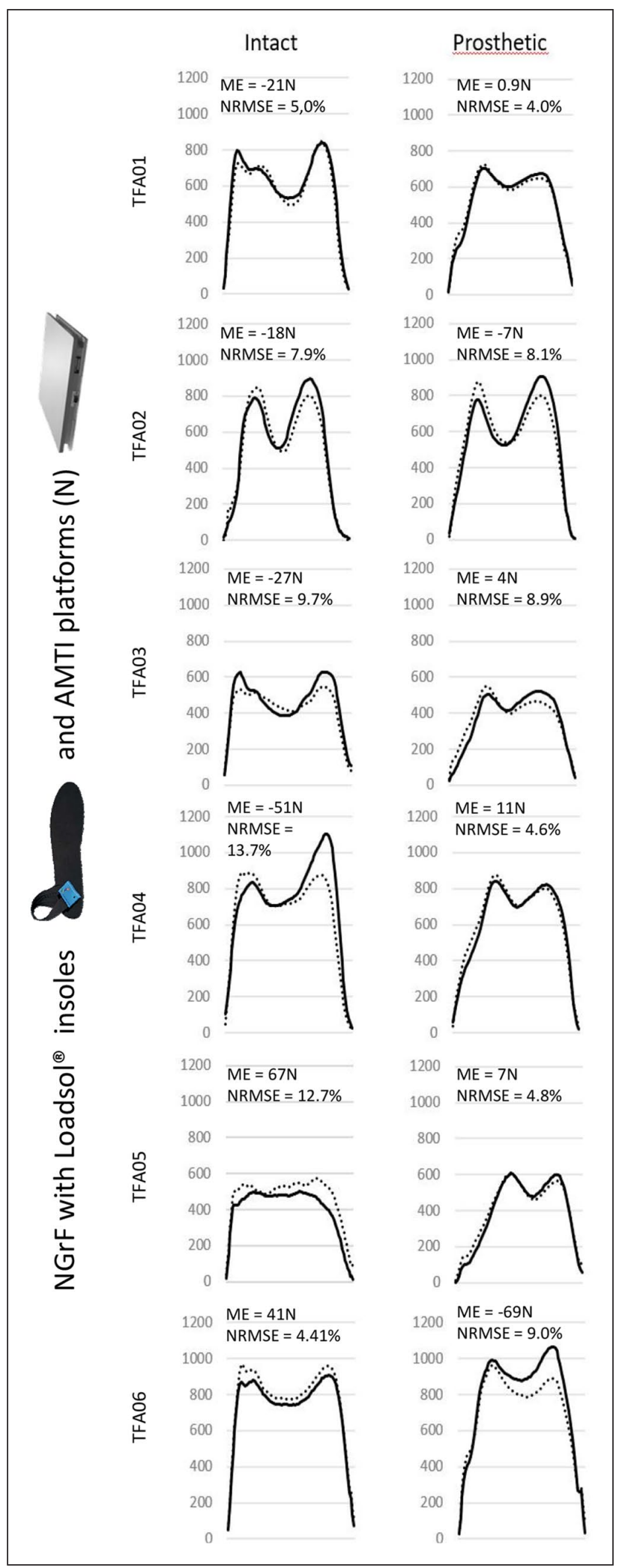

Figure 2. NGrF for intact and prosthetic limb of each TFA. The thick solid line corresponds to NGrF with Loadsol ${ }^{\circledR}$ insoles and the dotted line corresponds to $\mathrm{NGrF}$ with $A M T{ }^{\circledR}$ force plates. Mean error (ME) in Newton and normalized RMSE (NRMSE) (\%) obtained via insoles and that obtained by force plate for each amputee (TFAOI-TFA06).

\section{Results}

\section{Validation of Loadso ${ }^{\circledR}$ insoles}

The results show consistent similarities in NGrF data between the two systems $\left(\right.$ Loadsol $^{\circledR}$ insoles and AMTI ${ }^{\circledR}$ force platforms) for the six subjects for both the prosthetic and contralateral limbs (Figure 2). Pearson's coefficient was excellent for both sides ( 0.91 and 0.95 for the intact limb and prosthetic limb, respectively). The mean NRMSE was $6.6 \%$ (standard deviation $(\mathrm{SD})=2.3 \%$ ) for the prosthetic side and $8.9 \%(\mathrm{SD}=3.8 \%)$ for the intact side. The mean error (ME), which represents the bias between both measurement systems, was less than $69 \mathrm{~N}$ for the prosthetic side and less than $67 \mathrm{~N}$ for the intact side (Figure 2).

For the specific parameters (Fz1, Fz2, impulse, and stance phase) and their corresponding ASI values, the Bland-Altman plots (Figure 3) for both the prosthetic and the intact limbs showed interindividual variations in accuracy. The average normalized mean error (NME) for the group of TFAs ranged from $-9.18 \%$ to $3.73 \%$. The highest ME was found for Fz2. The ME of the stance phase duration was close to 0 . The ASI ME for each parameter ranged from $-2.67 \%$ to $4.35 \%$.

\section{Gait asymmetry quantification}

Mean cadence was 43.2 strides per minute for LSS, 51.5 for SSS, and 59.6 for FSS. No significant difference was found for any of the parameters between the walking speed conditions. Walking speed had an impact on NGrF ASI parameters. Loading asymmetry increased with walking speed.

Figure 4 depicts ASI parameter results for each TFA based on cadence. Concerning Fz1 asymmetry, five subjects had negative Fz1 ASI values at LSS and four subjects, at SSS, corresponding to a higher Fz1 value for the prosthetic limb than for the intact limb. At FSS, five subjects had positive Fz1 ASI values with a maximum of $35.85 \%$ ASI, suggesting that the force was higher on the intact limb than on the prosthetic limb. Maximum ASI values were obtained for Fz2 with $44.68 \%$ at FSS. Almost all subjects had positive ASI impulse values, ranging from $-0.97 \%$ to $38.41 \%$, regardless of the walking speed. Only one patient (TFA02) showed an ASI value lower than $11 \%$ for all parameters, regardless of cadence.

\section{Discussion}

\section{Loadso ${ }^{\circledR}$ validation}

The first aim of this study was to assess the validity of Loadsol ${ }^{\circledR}$ insoles to quantify NGrF during gait in people with transfemoral amputation. Loadsol ${ }^{\circledR}$ insoles were compared to force platforms considered as a gold standard. 


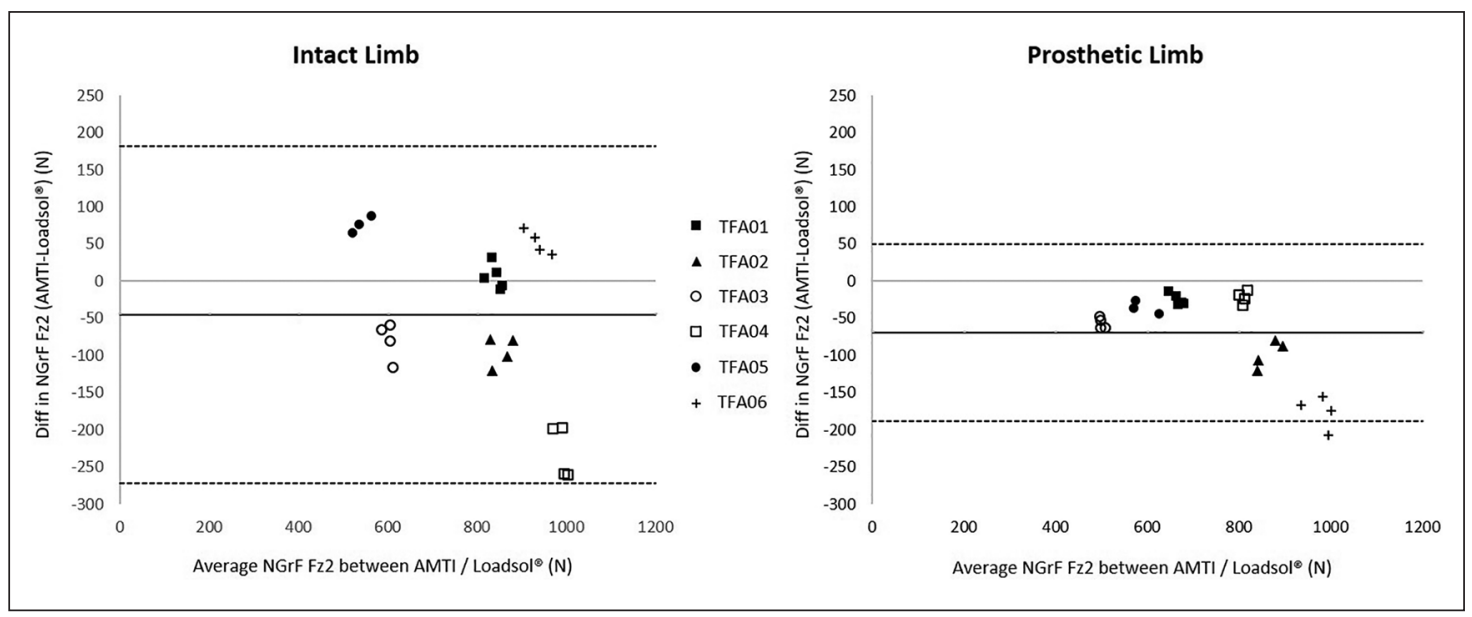

Figure 3. Bland-Altman plots for Fz2 (N) for intact and prosthetic side for each trial across participants. The thick line corresponds to the mean error and dotted line corresponds to the mean error \pm 2 SD.

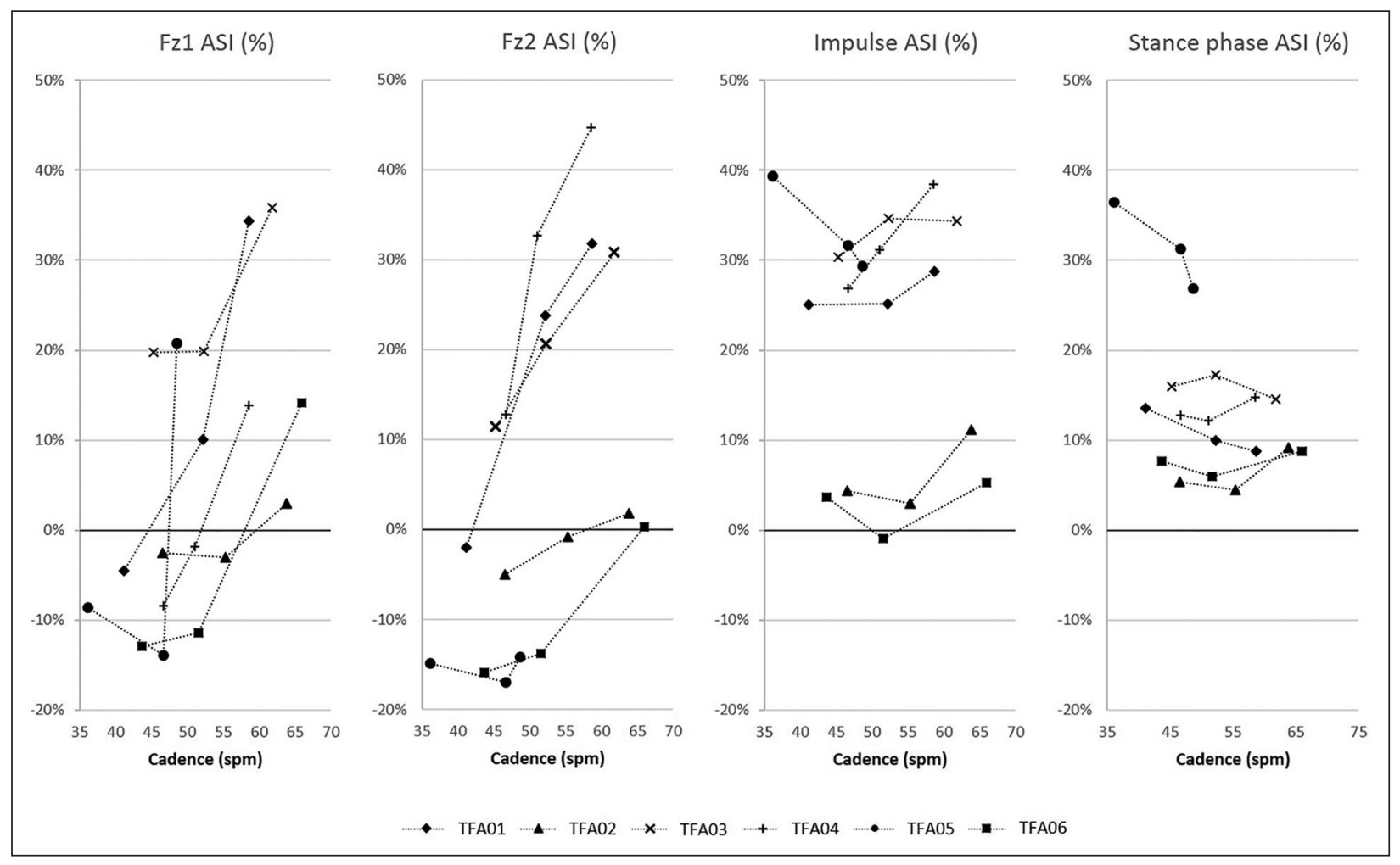

Figure 4. ASI (\%) for each parameter (FzI, Fz2, impulse, and stance phase) for each patient (TFA0I-TFA06) depending on cadence in strides per minute (spm).

Bland-Altman ${ }^{20}$ plots were used to characterize the consistency of both measurement systems for NGrF and asymmetry quantification. Our results support the validity of Loadsol ${ }^{\mathbb{B}}$ insoles with a mean difference of $-1.70 \mathrm{~N}$ for the intact limb and $-8.56 \mathrm{~N}$ for the prosthetic limb. The validity of this approach has already been investigated by Burns et al. ${ }^{21}$ on a population of 13 healthy subjects walking on a treadmill, resulting in a mean difference of $44.2 \mathrm{~N}$ when considering both feet. Complementary metrics (NME and NRMSE) were also consistent with studies in the literature concerning other insoles. 22,23

It must be noted that no previous study focused on the validation of insoles during gait in people with amputations except the one by Agrawal et al. ${ }^{24,25}$ The authors used F-Scan ${ }^{\circledR}$ sensors (Tekscan Inc., Boston, USA) with five 
people with transtibial amputations but did not report on the ME or the NRMSE.

Inspection of the Bland-Altman plots highlighted the interindividual variability of NGrF accuracy and sometimes revealed an overestimation of force values compared to the $\mathrm{AMTI}^{\circledR}$ force plates. This overestimation was not reported in the validation studies done with other insoles. ${ }^{6,26}$ The overestimated values could be explained by the sensitivity to the calibration procedure, which has already been shown by De Berardinis et $a .^{26}$ for Medilogic ${ }^{\circledR}$ insoles in healthy subjects. Another source of this discrepancy may lie in the difference in temperature inside the shoe between the prosthetic and contralateral limb, given that temperature is known to have an impact on measures. ${ }^{27}$ Individuals with transfemoral amputations can have difficulties in fully loading their prosthetic limb due to a lack of balance (loss of joints and muscles) and proprioception. To improve calibration, the therapist could perform the procedure using his own shoes before inserting the insoles in the patient's shoes. Another possibility would be to conduct independent and individual post-processing. ${ }^{28}$

\section{Gait asymmetry quantification}

The second aim of the study was to assess gait asymmetry in a group of TFAs on level ground at different walking speeds. In the present study, the index proposed by Nolan et al. ${ }^{6}$ was chosen to quantify the asymmetry of gait parameters. However, it must be pointed out that no consensus exists in the literature. To compare our results at SSS to the ones revealed by Cutti et al., ${ }^{8}$ the authors converted their results according to our indexes. All our results fall within the intervals defined by Cutti et al. for the asymmetry of stance duration (median: 10.4\%, 25th percentile: $4.9 \%$, 75 th percentile: $14 \%$ ), first peak in NGrF (median: $-2 \%$, 25th percentile: $-22.2 \%$, 75 th percentile: $3.9 \%$ ), and impulse (median: $14.8 \%$, 25th percentile: $0 \%$, 75th percentile: $21.4 \%$ ) reported for people equipped with (MPK). Our results also confirm that an overloading of the prosthetic limb can be observed at SSS. However, analysis at FSS highlighted individual differences that could not be observed at SSS. In particular, ASI values for cadence increased, especially at FSS, which is consistent with previous results in the literature. $5,6,24,25$ In addition, this speed condition resulted in greater forces on the intact limb than on the prosthetic limb, potentially increasing the risk of degenerative pathologies. Incidence of pain and osteoarthritis in the contralateral limb is known to be correlated to the overloading of the contralateral limb. ${ }^{11}$

The results also show interindividual variability of the parameters used to quantify the asymmetry in the group of TFAs. Thus, such parameters seem sensitive to individual ability and could be used to discriminate TFAs regarding asymmetry as shown in Figure 4. At FSS, the symmetry is very difficult to maintain, except for TFA02. This subject had an ASI value under $11.2 \%$ for each ASI parameter even at FSS, which is close to the ASI values observed in able bodies. ${ }^{6}$ These results prove that amputees can walk with minor loading asymmetry with a prosthesis with an MPK and a K3 prosthetic foot. The differences observed between amputees demonstrated the importance of the quantification of asymmetry during rehabilitation.

\section{Limitations}

This study presents several limitations. The overall number of amputees was limited but showed the importance of testing several walking speeds as individual differences can be highlighted as this analysis revealed. In addition, similar to Tekscan $^{\circledR 24}$ and Pedar ${ }^{\circledR}$ insoles, ${ }^{28}$ Loadsol $^{\circledR}$ insoles only measures the "normal component of the ground reaction" force. It does not measure shear forces. However, the vertical force corresponds to more than $90 \%$ of the resultant force during walking. ${ }^{12}$ Long-term recordings have not been performed compared to those performed in healthy subjects with Pedar ${ }^{\circledR 28}$ and Medilogic ${ }^{\circledR}$ sensors. ${ }^{22}$ The possible drift of the insoles as Hurkmans et al. ${ }^{28}$ observed with the Pedar ${ }^{\circledR}$ system should be taken into account. The authors propose a drift correction algorithm to integrate a zero setting after $1 \mathrm{~h}$ of usage in the protocol.

Furthermore, insole force measurements may have been influenced by the type of footwear. ${ }^{23}$ Our participants wore their own shoes rather than standardized shoes. Moreover, two participants (TFA01 and TFA02) wore therapeutic insoles on the intact side. Thus, Loadsol insoles were positioned between the therapeutic insole and the shoe, which could have enhanced the stiffness of the shoe and affected the results.

Finally, further studies are necessary to evaluate different calibrations for amputees, between-day repeatability, influence of long-term recordings, and particularly, the possibility of drift.

\section{Conclusion}

The present prospective study quantified the validity of Loadsol ${ }^{\mathbb{R}}$ insoles compared to force platforms and the possibility to assess loading asymmetry during gait in TFAs. The study highlights the effect of walking speed on asymmetry. Gait analysis at fast speed revealed some differences between individuals. After correcting the calibration problem described above and verifying the occurrence of drift, this device, which is "clinically easy to use," could be beneficial for both researchers and clinicians. This system shows evidence of beneficial application in the rehabilitation and follow-up of patients with lower limb deficiencies (amputation and fractures) by providing the proportion of force transmitted by each lower limb using a vibrating or 
audio feedback system. Performance (evaluation during real-life activities) and capacity (evaluation in a laboratory) could be assessed through long-term recordings. Moreover, real-time NGrF feedback opens perspectives for rehabilitation techniques. Auto-rehabilitation between rehabilitation sessions could be also considered and should be very useful in enhancing recovery of gait symmetry.

\section{Acknowledgements}

The authors would like to express their gratitude to the IRR center and the INI center for prosthesis fitting. They also acknowledge the essential contributions of Marie-Agnès Haldric for the technical support, Florian Guillaume for the technical discussions, Julia Facione for the recruitment of transfemoral amputees, and Jonathan Pierret for his statistical support. The study was performed in Institut de Biomécanique Humaine Georges Charpak, Arts et Metiers ParisTech, Paris, France, and Centre de médecine physique et de réadaptation Louis Pierquin, IRRUGECAM, Nancy Cedex, France.

\section{Author contribution}

All authors contributed equally in the preparation of this manuscript.

\section{Declaration of conflicting interests}

The author(s) declared the following potential conflicts of interest with respect to the research, authorship, and/or publication of this article: We certify that no party having a direct interest in the results of the research supporting this article has or will confer a benefit on us or on any organization with which we are associated, and we certify that all financial and material support for this research and work are clearly identified by each author's institution (Isabelle Loiret, Coralie Villa, Boris Dauriac, Xavier Bonnet, Noël Martinet, Jean Paysant, and Hélène Pillet).

\section{Funding}

The author(s) received no financial support for the research, authorship, and/or publication of this article.

\section{ORCID iD}

Isabelle Loiret iD https://orcid.org/0000-0001-6990-9982

\section{References}

1. Drevelle X, Villa C, Bonnet X, et al. Vaulting quantification during level walking of transfemoral amputees. Clin Biomech 2014; 29(6): 679-683.

2. Esquenazi A. Gait analysis in lower-limb amputation and prosthetic rehabilitation. Phys Med Rehabil Clin N Am 2014; 25(1): 153-167.

3. Cappozzo A, Figura F, Gazzani F, et al. Angular displacements in the upper body of AK amputees during level walking. Prosthet Orthot Int 1982; 6(3): 131-138.

4. Villa C, Drevelle X, Bonnet X, et al. Evolution of vaulting strategy during locomotion of individuals with transfemoral amputation on slopes and cross-slopes compared to level walking. Clin Biomech 2015; 30(6): 623-628.
5. Nolan L and Lees A. The functional demands on the intact limb during walking for active trans-femoral and trans-tibial amputees. Prosthet Orthot Int 2000; 24(2): 117-125.

6. Nolan L, Wit A, Dudzinski K, et al. Adjustments in gait symmetry with walking speed in TF and TT amputee. Gait Posture 2003; 17: 142-151.

7. Schaarschmidt M, Lipfert SW, Meier-Gratz C, et al. Functional gait asymmetry of unilateral transfemoral amputees. Hum Mov Sci 2012; 31(4): 907-917.

8. Cutti AG, Verni G, Migliore GL, et al. Reference values for gait temporal and loading symmetry of lower-limb amputees can help in refocusing rehabilitation targets. $J$ Neuroeng Rehabil 2018; 15(Suppl. 1): 61.

9. Rutkowska-Kucharska A, Kowal M and Winiarski S. Relationship between asymmetry of gait and muscle torque in patients after unilateral transfemoral amputation. Appl Bionics Biomech 2018; 2018: 1-9.

10. Struyf PA, vanHeugten CM, Hitters MW, et al. The prevalence of osteoarthritis of the intact hip and knee. Arch Phys Med Rehabil 2009; 90(3): 440-446.

11. Gailey R, Allen K, Castles J, et al. Review of secondary physical conditions associated with lower-limb amputation and long-term prosthesis use. J Rehabil Res Dev 2008; 45(1): 15-29.

12. Price C, Parker D and Nester C. Validity and repeatability of three in-shoe pressure measurement systems. Gait Posture 2016; 46: 69-74.

13. Hsiao H, Guan J and Weatherly M. Accuracy and precision of two in-shoe pressure measurement systems. Ergonomics 2002; 45(8): 537-555

14. Putti AB, Arnold GP, Cochrane L, et al. The Pedar®inshoe system: repeatability and normal pressure values. Gait Posture 2007; 25(3): 401-405.

15. Ramanathan AK, Kiran P, Arnold GP, et al. Repeatability of the Pedar-X®in-shoe pressure measuring system. Foot Ankle Surg 2010; 16(2): 70-73.

16. Braun BJ, Veith NT, Hell R, et al. Validation and reliability testing of a new, fully integrated gait analysis insole. J Foot Ankle Res 2015; 8: 54-57.

17. Tang UH, Zugner R, Lisovskaja V, et al. Foot deformities, function in the lower extremities, and plantar pressure in patients with diabetes at high risk to develop foot ulcers. Diabet Foot Ankle 2015; 6: 27593.

18. Chopra S, Rouhani H, Assal M, et al. Outcome of unilateral ankle arthrodesis and total ankle replacement in terms of bilateral gait mechanics. J Orthop Res 2014; 32(3): 377-384

19. Mariani B, Rouhani H, Crevoisier X, et al. Quantitative estimation of foot-flat and stance phase of gait using foot-worn inertial sensors. Gait Posture 2013; 37(2): 229-234.

20. Bland JM and Altman DG. Statistical methods for assessing agreement between two methods of clinical measurement. Lancet 1986; 1(8476): 307-310.

21. Burns GT, Deneweth Zendler J and Zernicke RF. Validation of a wireless shoe insole for ground reaction force measurement. J Sports Sci 2019; 37(10): 1129-1138.

22. Koch M, Lunde LK, Ernst M, et al. Validity and reliability of pressure-measurement insoles for vertical ground reaction force assessment in field situations. Appl Ergon 2016; 53(Pt. A): 44-51. 
23. Barnett S, Cunningham JL and West S. A Comparison of vertical force and temporal parameters produced by an in-shoe pressure measuring system and a force platform. Clin Biomech 2001; 16: 353-357.

24. Agrawal V, Gailey RS, Gaunaurd IA, et al. Comparison of four different categories of prosthetic feet during ramp ambulation in unilateral transtibial amputees. Prosthet Orthot Int 2015; 39(5): 380-389.

25. Agrawal V, Gailey R, Gaunaurd IA, et al. Comparison between microprocessor-controlled ankle/foot and conventional prosthetic feet during stair negotiation in people with unilateral transtibial amputation. J Rehabil Res Dev 2013; 50(7): 941-950.
26. De Berardinis J, Dufek JS, Trabia MB, et al. Assessing the validity of pressure-measuring insoles in quantifying gait variables. J Rehabil Assist Technol Eng 2018; 5: DOI: 10.1177/2055668317752088.

27. Herbert-Copley AG, Sinitski EH, Lemaire ED, et al. Temperature and measurement changes over time for F-Scan sensors. In: Proceedings of the 2013 IEEE international symposium on medical measurements and applications (MeMeA), Gatineau, QC, Canada, 4-5 May 2013, pp. 265-267. New York: IEEE.

28. Hurkmans HLP, Bussmann JBJ, Selles RW, et al. Validity of the Pedar mobile system for vertical force measurement during a seven-hour period. J Biomech 2006; 39(1): 110-118. 